\title{
Kleinpapulöse Hautsarkoidose bei einem Ehepaar
}

\author{
B. Knopf ${ }^{1}$ \\ L.-W. Nali \\ A. Tannapfel ${ }^{3}$
}

\section{Small-Node Cutaneous Sarcoidosis in a Married Couple}

\section{Zusammenfassung}

Es wird über ein Ehepaar berichtet, das zeitgleich an einer kleinpapulösen kutanen Sarkoidose erkrankte. Klinik und Histologie stimmten in beiden Fällen überein. Außer erhöhten IgG-Antikörpern gegen CMV und HSV gab es in den Laborbefunden keine Besonderheiten. Mykobakterielle Strukturen konnten mittels PCRTechnik nicht nachgewiesen werden. Unter kortisonhaltigen Externa sowie einer Antibiose mit Doxycyclin heilten die Hautherde langsam ab. Die Hypothese einer infektiösen Genese der Sarkoidose wird durch diese Fälle aus epidemiologischer Sicht unterstützt.

\section{Abstract}

We report a married couple which has been taken ill with a small-node cutaneous sarcoidosis at the same time. Clinical signs and histology corresponded in both cases. Except increased IgG antibodies against CMV and HSV there were no characteristics in the laboratory results. No mycobacterial structures could be found by polymerase chain reaction (PCR). With external corticosteroids and an antibiotic therapy with doxycycline the skin lesions healed up slowly. From epidemiological point of view the hypothesis of an infectious genesis of sarcoidosis is being supported by these cases.

\section{Einleitung}

Die Sarkoidose ist eine granulomatöse Multisystemerkrankung.

Die Haut kann sowohl als isolierte Hautsarkoidose als auch in etwa einem Drittel der Fälle im Rahmen einer Systemerkrankung befallen sein.

Die Ätiologie der Sarkoidose ist bis heute nicht geklärt. Eine Reaktion auf unbekannte infektiöse Antigene oder Autoantigene bei entsprechender genetischer Disposition wird diskutiert.

Trotz vielfältiger Bemühungen konnte die infektiöse Genese jedoch bisher weder bewiesen noch bestätigt werden.
Vor allem epidemiologische Daten gaben Hinweise auf eine mögliche Infektionshypothese. Unsere beiden Fälle, nämlich das Auftreten einer Hautsarkoidose zeitgleich bei einem Ehepaar, unterstützen die Hypothese.

\section{Fallberichte}

\section{Kasuistik 1}

Anamnese

B. Ch.: 53 Jahre, weiblich, Schneiderin

Seit 11/01 entstanden an den Unterarmen, Oberschenkeln, Unterschenkeln kleine rote Knötchen ohne subjektive Beschwerden.

${ }^{1}$ Klinik für Hautkrankheiten und Allergologie, Städtisches Klinikum Zwickau

${ }^{2}$ Hautarztpraxis Zwickau

${ }^{3}$ Institut für Pathologie der Universität Leipzig 


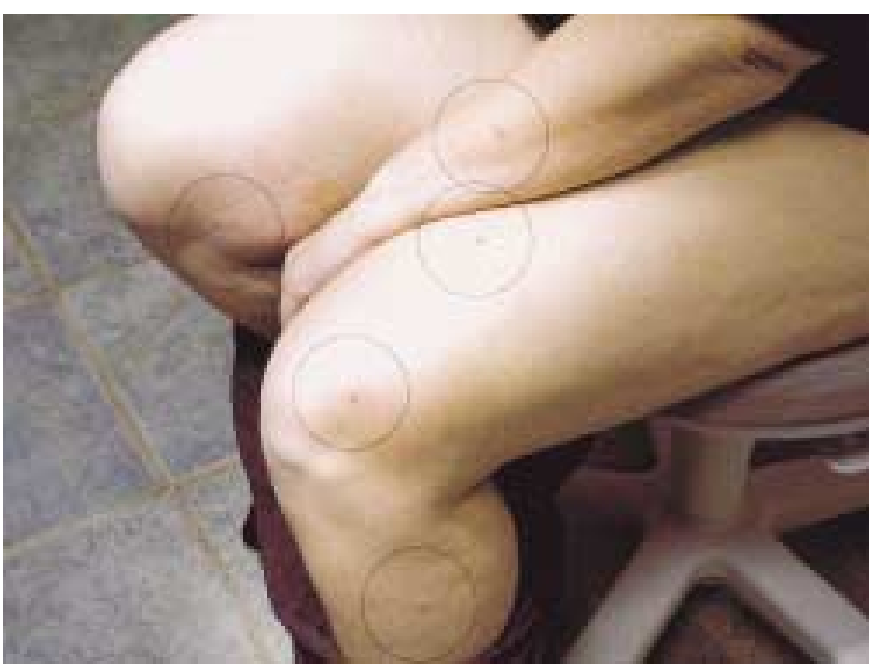

Abb. 1 Sarkoidoseknötchen Oberschenkel von Pat. B., Ch. (Fernansicht).

Ähnliche Hautveränderungen traten zeitgleich beim Ehemann (B. H.-M., Kasuistik 2) auf.

Hautbefund (10.12.01)

An beiden Unterarmen, beiden Oberschenkeln, Knien, linker Unterschenkelaußenseite bestehen mäßig gerötete, derbe, linsengroße Papeln (Abb.1).

\section{Dermatohistopathologie (E-Nr. 32 944/01)}

Man erkennt mehrschichtiges hyperplastisches Plattenepithel der Haut, im subepithelialen Bindegewebe eine geringe lymphozytäre Entzündungsreaktion, die lymphozytären Zellelemente teilweise granulomartige Herde umschließend. Innerhalb der Granulome finden sich Epitheloidzellen, teilweise histiozytäre Zellelemente (Abb.2a,b). PCR im Biopsiematerial: kein Nachweis (atypischer) Mykobakterien.

\section{Laborbefunde}

Routinelaborparameter im Normbereich.

Virusserologie: CMV-AK (IgG) 13000 U/ml erhöht.

HSV-AK (IgG) 16000 U/ml erhöht.

CMV-AK (IgM), HSV-AK (IgM), EBV, HHV 8: negativ.

TPHA, Borrelienserologie, ANF, Kalzium, ACE, Neopterin, s-IL2-Rezeptor: unauffällig.

\section{Weitere Befunde}

Tuberkulintest (10 TE): positiv.

Röntgen-Thorax: kein Hinweis auf Sarkoidose.

Oberbauchsonographie: unauffällig.

\section{Verlauf}

Unter kortisonhaltigen Externa und $2 \times 100 \mathrm{mg}$ Doxycyclin/d langsame Abheilung.

\section{Kasuistik 2 \\ Anamnese}

B. H.-M.: 51 Jahre, männlich, Schlosser

Seit 11/01 entwickelten sich symptomlose rötliche Knötchen an beiden Oberschenkeln (Abb. 3) und der linken Schläfe.

Der Patient kann keine Angaben zur Ursache machen. Ähnliche Hautveränderungen traten zeitgleich bei der Ehefrau (B. Ch., Kasuistik 1) auf.

\section{Hautbefund (10.12.01)}

An beiden Oberschenkelstreckseiten und -beugeseiten sowie an der linken Schläfe bestehen linsengroße, intensiv gerötete bis lividrote, derbe Papeln (Abb. 3).
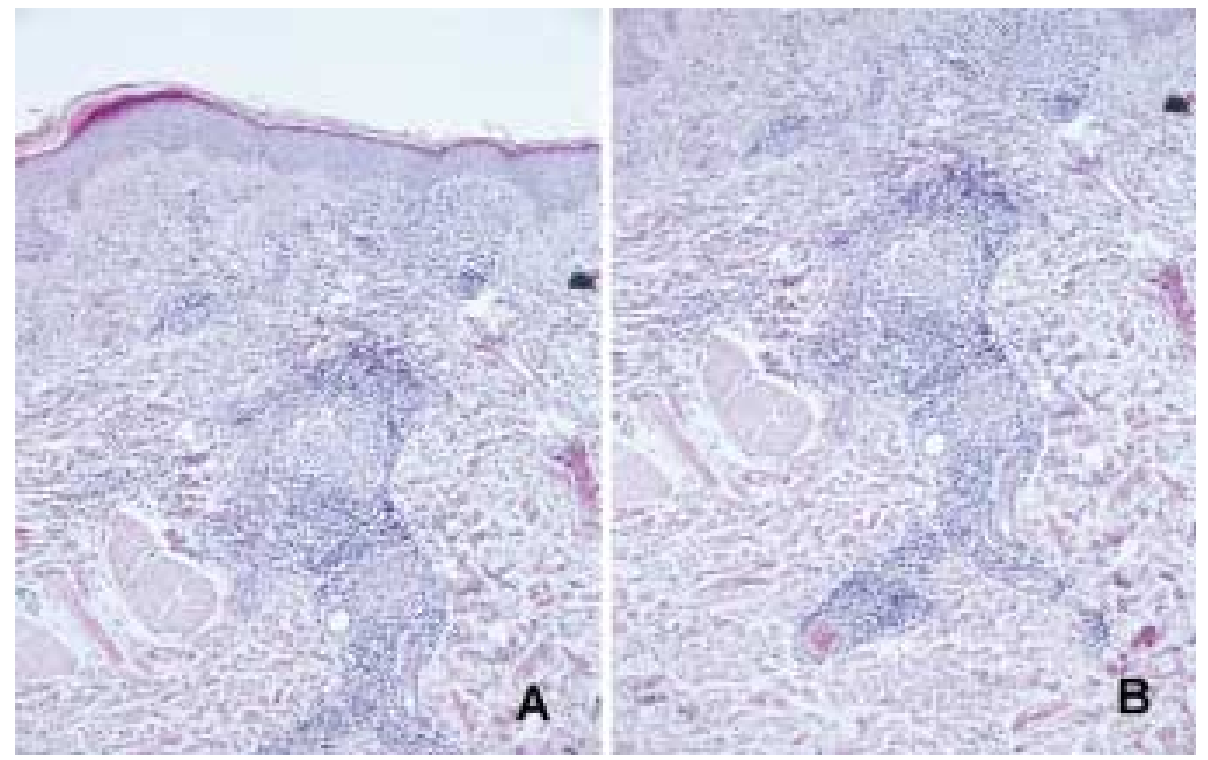

Abb. 2a,b Sarkoidoseherde in der Haut Epitheloidzellige Granulome mit einzelnen Langhans-Riesenzellen und einem schmalen Lymphozytensaum. (a) Hämatoxylin-Eosin, Originalvergrößerung 15fach. (b) Hämatoxylin-Eosin, Originalvergrößerung 40fach. 


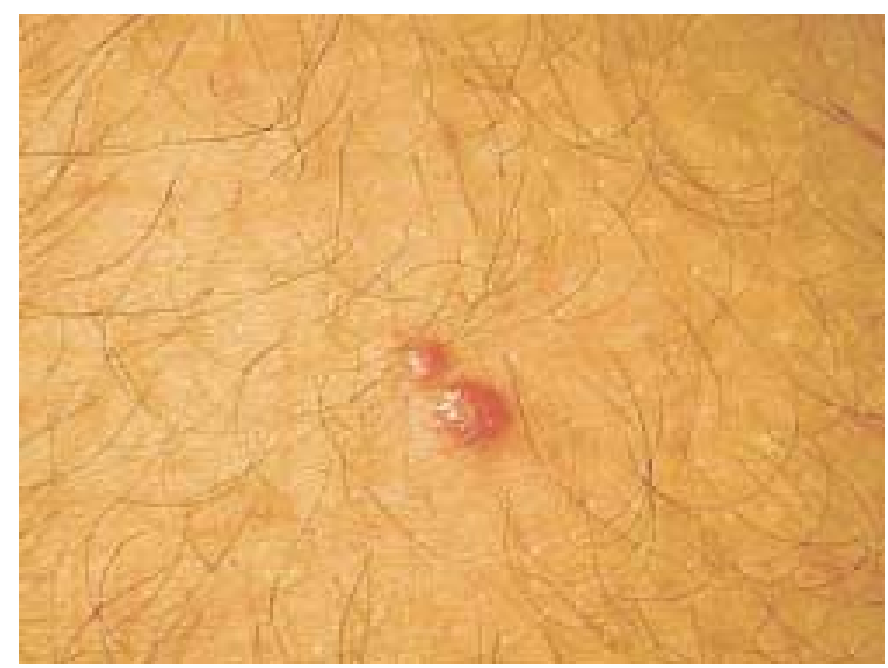

Abb. 3 Sarkoidoseknötchen am Oberschenkel von Pat. B., H.-M. (Nahaufnahme).

\section{Dermatohistopathologie (E-Nr. 32 945/01)}

Man erkennt mehrschichtiges, hyperplastisches Plattenepithel der Haut. Die Hautanhangsgebilde sind regelrecht. Im subepithelialen Bindegewebe zeigen sich bei Aufarbeitung in Schnittstufen lymphozytäre Zellinfiltrate, diese teilweise Epitheloidzellen umschließend, diese mit flachen, teilweise schuhsohlenartigen Kernformationen, z.T. kommen granulomartige Bindegewebsneubildungen zur Darstellung (Abb. 4a,b,c).

PCR im Biopsiematerial: kein Nachweis (atypischer) Mykobakterien.

\section{Laborbefunde}

Routinelaborparameter im Normbereich.

Virusserologie: CMV-AK (IgG) 3200 U/ml erhöht.
HSV-AK (IgG) 13000 U/ml erhöht.

CMV-AK (IgM), HSV-AK (IgM), EBV, HHV8: negativ.

TPHA, Borrelienserologie, ANF, Kalzium, ACE, Neopterin, s-IL2-Rezeptor: unauffällig.

\section{Weitere Befunde}

Tuberkulintest (10 TE): positiv.

Röntgen-Thorax: kein Hinweis auf Sarkoidose.

Oberbauchsonographie: unauffällig.

\section{Verlauf}

Unter kortisonhaltigen Externa und $2 \times 100 \mathrm{mg}$ Doxycyclin/d langsame Rückbildung der Hautveränderungen.

\section{Epidemiologische Anamnese}

Das Ehepaar lebt allein. Die beiden erwachsenen Kinder, die Eltern und die Geschwister haben einen anderen Wohnsitz. Weder die Verwandten noch die Arbeitskollegen zeigten ähnliche Hautveränderungen.

\section{Diskussion}

Bereits 1905 vermutete C. Boeck, dass es sich bei der Sarkoidose um eine bakterielle Infektionskrankheit handeln könnte.

Über die Stimulation zellulärer Immunmechanismen, z. B. durch bakterielle Antigene und bei entsprechender genetischer Disposition, wären die Entstehung der typischen granulomatösen Veränderungen einer Sarkoidose denkbar.

Epidemiologische Beobachtungen, wie z.B. erhöhte Sarkoidoseprävalenz bei Krankenhauspersonal, jahreszeitliche und lokale Häufigkeiten, gehäuftes familiäres Auftreten und auch Hinweise
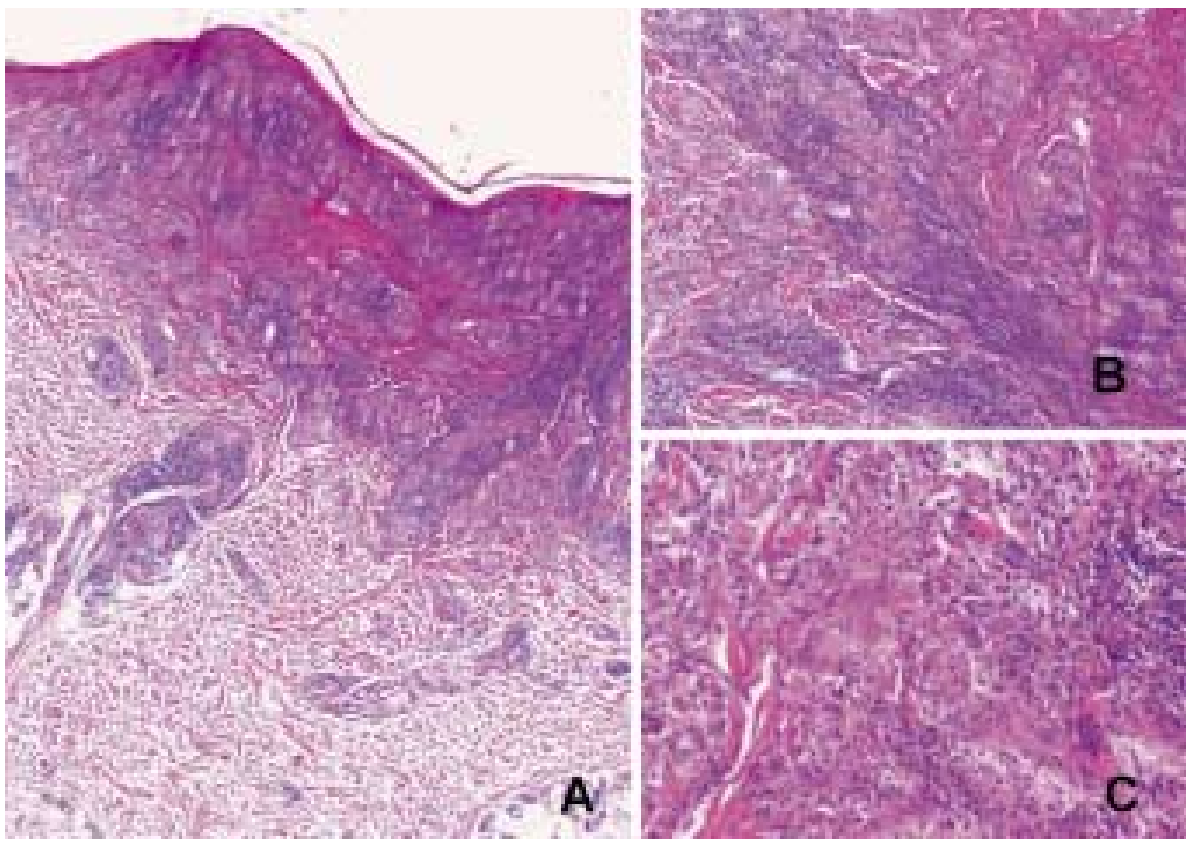

Abb. 4a,b,c Sarkoidoseherde im Corium und Subkutangewebe der Haut mit Epitheloidzellen, Langhans-Riesenzellen (b und $\mathbf{c}$ ) und einem deutlichen Lymphozyteninfiltrat (b). Die Granulome sind von einem retikulären, entzündlich durchsetzten Bindegewebe umgeben (c). 
aus der Transplantationsmedizin unterstützen die Infektionshypothese.

Durch den Nachweis von mykobakterieller DNA oder mykobakterieller RNA in sarkoidalen Granulomen mittels PCR-Technik erhielt die infektiöse Genese zusätzlichen Auftrieb [1 -3].

Auch Li et al. [4] wiesen in 16 von 20 Fällen mit Sarkoidose mykobakterielle DNA mittels PCR-Technik nach. Ihnen gelang mit Hilfe der PCR/REPA-Technik in 2 Fällen der Nachweis von M. tuberculosis complex, in 4 Fällen M. avium und in 2 Fällen M. kausasii. Die anderen 8 Fälle konnten nicht näher bestimmt werden.

Von anderen Untersuchern wurden diese Befunde wiederum nicht bestätigt $[5,6]$.

Interessant ist in diesem Zusammenhang die Arbeit von ToutousTrellu et al. [7], die 3 Fälle von Hautsarkoidose mit Clarithromycin $1 \mathrm{~g} / \mathrm{d}$ und Ciprofloxacin $1 \mathrm{~g} / \mathrm{d}$ über 6 Monate behandelten.

Vor und nach der Antibiose wurde in Hautschnitten nach bakterieller DNA und $16 \mathrm{~S}$ ribosomalen RNA-Sequenzen gesucht. Die Amplifikation für bakterielle DNA war in allen Hautbiopsien positiv. Eine Sequenzierung der DNA hinsichtlich bakteriellen Spezies gelang jedoch nicht. Eine Abheilung unter der Antibiose trat ebenfalls nicht ein.

Neben der Vermutung, dass M. tuberculosis in der Ätiologie der Sarkoidose eine Rolle spielt, werden auch Borrelia burgdorferi [8], Chlamydia pneumoniae [9] und Propionibakterien [10] als möglicher Auslöser oder Kofaktoren diskutiert.

Alberti et al. [11] berichteten über den Nachweis von humanem Herpes-Virus-Typ 8 (HHV-8) im Sarkoidosegewebe. Regamey et al. [12] sowie Sugaya et al. [13] konnten diese Befunde allerdings nicht bestätigen.

Die Aufzählung solcher widersprüchlicher Ergebnisse könnte fortgeführt werden. Ursache für diese Unterschiede dürfte sicherlich die Schwierigkeit sein, mit molekularbiologischen Methoden bekannte oder unbekannte Erreger im Gewebe zweifelsfrei zu identifizieren.

Auf die mögliche Auslösung der Sarkoidose durch unbelebte Erreger (Umwelteinfluss) soll nur hingewiesen, jedoch nicht näher in diesem Zusammenhang eingegangen werden.

Nach heutigen Kenntnissen ist es bisher nicht gelungen, Auslöser der Sarkoidose zu identifizieren und davon entsprechende therapeutische Konsequenzen abzuleiten [14].

Die infektiöse Hypothese der Sarkoidose ist somit, wie bereits eingangs erwähnt, weder zu bejahen noch zu verneinen.
Bei unserem Ehepaar fielen in der Serologie bei beiden erhöhte IgG-Antikörper gegen CMV und HSV auf. Einen ätiologischen Zusammenhang zur Sarkoidose zu sehen, erscheint unwahrscheinlich.

Die Behandlung mit Doxycyclin erfolgte ex juvantibus unter der Vorstellung, dass eine infektiöse Genese aus rein epidemiologischen Gründen vorliegt. Der Nachweis von (atypischen) Mykobakterien mittels PCR-Technik gelang jedoch nicht.

Die langsame Abheilung unter einer kortisonhaltigen Salbe sowie Doxycyclin kann man nicht als zwingendes Argument für die infektiöse Genese ansehen. Spontane Abheilungen sind bei der Sarkoidose nicht selten.

Letztendlich bleibt nur die bemerkenswerte Tatsache des zeitgleichen Auftretens einer kutanen Sarkoidose bei einem Ehepaar.

Die Infektionshypothese der Sarkoidose könnte dadurch zumindest aus epidemiologischer Sicht gestützt werden.

\section{Literatur}

${ }^{1}$ Grosser M, Luther T, Müller J, Schuppler J, Bickhardt W, Matthiessen W, Müller M. Detection of M. tuberculosis DNA in sarcoidosis: correlation with T-cell response. Lab Invest 1999; 79: 775

2 Popper HH, Klemm H, Hoefler G, Winter E. Presence of mycobacterial DNA in sarkoidosis. Hum Pathol 1979; 28: 796

${ }^{3}$ Degitz K. Detection of mycobacterial DNA in the skin. Etiologic insights and diagnostic perspectives. Arch Dermatol 1996; 132: 71 - 75

${ }^{4}$ Li N, Bagoghli A, Kubba K, Bhawan J. Identification of mycobacterial DNA in cutaneous lesions of sarcoidosis. J Cutan Pathol 1999; 26: $271-278$

${ }^{5}$ Wilsher ML, Menzies RE, Croxon MC. Mycobacterium tuberculosis DNA in tissue affected by sarcoidosis. Thorax 1998; 53: 871

${ }^{6}$ Vokurka M, Lecossier D, de Bois RM, Wallaert B, Kambuchner M, Tazi A, Hance AJ. Absence of DNA from mycobacteria of the M. tuberculosis complex in sarcoidosis. Am J Respir Crit Car Med 1997; 156: 1000

7 Toutous-Trellu L, Ninef B, Rohner P, Auckentaler R, Sanrat JH, Pechere M. Three cases of cutaneous sarcoidosis: search for bacterial agent by the 16 S RNA gene analysis and treatment with antibiotics. Dermatology 2000; 4: $242-245$

${ }^{8}$ Gruber TA, Eganzuddin S. Positive Lyme titers in a patient with active sarcoidosis. Sarcoid Vasult Diffuse Lung Dis 1997; 14: 86

9 Puolakkainen M, Campbell LA, Kuo CC, Leinonen M, Grönhagen-Riska C, Saikka P. Serological response to Chlamydia pneumoniae in patients with sarcoidosis. J Infection 1996; 33: 199

${ }^{10}$ Istrige J, Usui Y, Takemura T, Eishi Y. Quantitative PCR of mycobacterial and propionibacterial DNA in lymph node of Japanese patient with sarcoidosis. Lancet 1999; 354: 120

${ }^{11}$ Alberti LD, Piatelli A, Artese L et al. Human herpesvirus 8 variants in sarcoid tissue. Lancet 1997; 350: 1655-1661

${ }^{12}$ Regamey N, Erb P, Tamm M, Cathomas O. Human herpesvirus 8 variants. Lancet 1998; 351: 680

${ }^{13}$ Sugaya M, Nakamura K, Takahiro W, Tamaki K. Human herpesvirus typ 8 is not detected in cutaneous lesions of sarcoidosis. Brit J Dermatol 1999; 141: 769

${ }^{14}$ Müller-Querheim J. Die Ätiologie der Sarkoidose: Eine Suche nach belebten und unbelebten Auslösern. In: Kirsten D, Magnussen H (Hrsg). Sarkoidose update 2000. Interpneu Verlag 